Article

\title{
Study on the Development of Neutrosophic Triplet Ring and Neutrosophic Triplet Field
}

\author{
Mumtaz Ali ${ }^{1}{ }^{(\mathbb{B}}$, Florentin Smarandache ${ }^{2, *}$ (i) and Mohsin Khan ${ }^{3}$ \\ 1 Department of Mathematics, Environment and Science, University of Southern Queensland, Springfield, \\ QLD 4300, Australia; Mumtaz.Ali@usq.edu.au \\ 2 Department of Mathematics, University of New Mexico, 705 Gurley Avenue, Gallup, NM 87301, USA \\ 3 Department of Mathematics, Abdul Wali Khan University, Mardan 23200, Pakistan; \\ mohsinkhan7284@gmail.com \\ * Correspondence: fsmarandache@gmail.com
}

Received: 19 February 2018; Accepted: 18 March 2018; Published: 23 March 2018

check for updates

\begin{abstract}
Rings and fields are significant algebraic structures in algebra and both of them are based on the group structure. In this paper, we attempt to extend the notion of a neutrosophic triplet group to a neutrosophic triplet ring and a neutrosophic triplet field. We introduce a neutrosophic triplet ring and study some of its basic properties. Further, we define the zero divisor, neutrosophic triplet subring, neutrosophic triplet ideal, nilpotent integral neutrosophic triplet domain, and neutrosophic triplet ring homomorphism. Finally, we introduce a neutrosophic triplet field.
\end{abstract}

Keywords: ring; field; neutrosophic triplet; neutrosophic triplet group; neutrosophic triplet ring; neutrosophic triplet field

\section{Introduction}

The concept of a ring first arose from attempts to prove Fermat's last theorem [1], starting with Richard Dedekind in the 1880s. After contributions from other fields, mainly number theory, the notion of a ring was generalized and firmly established during the 1920s by Emmy Noether and Wolfgang Krull [2]. Modern ring theory, a very active mathematical discipline, studies rings in their own right. To explore rings, mathematicians have devised various notions to break rings into smaller, more understandable pieces, such as ideals, quotient rings, and simple rings. In addition to these abstract properties, ring theorists also make various distinctions between the theories of commutative rings and noncommutative rings, the former belonging to algebraic number theory and algebraic geometry. A particularly rich theory has been developed for a certain special class of commutative rings, known as fields, which lies within the realm of field theory. Likewise, the corresponding theory for noncommutative rings, that of noncommutative division rings, constitutes an active research interest for noncommutative ring theorists. Since the discovery of a mysterious connection between noncommutative ring theory and geometry during the 1980s by Alain Connes [3-5], noncommutative geometry has become a particularly active discipline in ring theory.

The foundation of the subject (i.e., the mapping from subfields to subgroups and vice versa) is set up in the context of an absolutely general pair of fields. In addition to the clarification that normally accompanies such a generalization, there are useful applications to infinite algebraic extensions and to the Galois Theory of differential equations [6]. There is also a logical simplicity to the procedure: everything hinges on a pair of estimates of field degrees and subgroup indices. One might describe it as a further step in the Dedekind-Artin linearization [7].

An early contributor to the theory of noncommutative rings was the Scottish mathematician Wedderburn who, in 1905, proved "Wedderburn's Theorem", namely that every finite division ring is 
commutative and so is a field [8]. It was only around the 1930s that the theories of commutative and noncommutative rings came together and that their ideas began to influence each other.

Neutrosophy is a new branch of philosophy which studies the nature, origin, and scope of neutralities as well as their interaction with ideational spectra. The concept of neutrosophic logic and a neutrosophic set was first introduced by Florentin Smarandache [9] in 1995, where each proposition in neutrosophic logic is approximated to have the percentage of truth in a subset $\mathrm{T}$, the percentage of indeterminacy in a subset I, and the percentage of falsity in a subset F such that this neutrosophic logic is called an extension of fuzzy logic, especially to intuitionistic fuzzy logic [10]. The generalization of classical sets [9], fuzzy sets [11], and intuitionistic fuzzy sets [10], etc., is in fact the neutrosophic set. This mathematical tool is used to handle problems consisting of uncertainty, imprecision, indeterminacy, inconsistency, incompleteness, and falsity. By utilizing the idea of neutrosophic theory, Vasantha Kandasamy and Florentin Smarandache studied neutrosophic algebraic structures [12-14] by inserting a literal indeterminate element " $\mathrm{I}$ ", where $\mathrm{I}^{2}=\mathrm{I}$, in the algebraic structure and then combining " $\mathrm{I}$ " with each element of the structure with respect to the corresponding binary operation, denoted * They call it the neutrosophic indeterminate element, and the generated algebraic structure is then termed as a neutrosophic algebraic structure. Some other neutrosophic algebraic structures can be seen as neutrosophic fields [15], neutrosophic vector spaces [16], neutrosophic groups [17], neutrosophic bigroups [17], neutrosophic N-groups [15], neutrosophic semigroups [12], neutrosophic bisemigroups [12], neutrosophic N-semigroups [12], neutrosophic loops [12], neutrosophic biloops [12], neutrosophic N-loop [12], neutrosophic groupoids [12] and neutrosophic bigroupoids [12] and so on.

In this paper, we introduce the neutrosophic triplet ring. Further, we define the neutrosophic triplet zero divisor, neutrosophic triplet subring, neutrosophic triplet ideal, nilpotent neutrosophic triplet, integral neutrosophic triplet domain, and neutrosophic triplet ring homomorphism. Finally, we introduce a neutrosophic triplet field. The rest of the paper is organized as follows. After the literature review in Section 1 and basic concepts in Section 2, we introduce the neutrosophic triplet ring in Section 3. Section 4 is about the introduction of the integral neutrosophic triplet domain with some of its interesting properties, and is also where we develop the neutrosophic triplet ring homomorphism. In Section 5, we study neutrosophic triplet fields. Conclusions are given in Section 6.

\section{Basic Concepts}

In this section, all definitions and examples have been taken from [18] to provide some basic concepts about neutrosophic triplets and neutrosophic triplet groups.

Definition 1. Let $N$ be a set together with a binary operation *. Then $N$ is called a neutrosophic triplet set if for any $a \in N$, there exists a neutral of " $a$ " called neut $(a)$, different from the classical algebraic unitary element, and an opposite of " $a$ " called anti( $a)$, with neut $(a)$ and anti $(a)$ belonging to $N$, such that

$$
a * \operatorname{neut}(a)=\operatorname{neut}(a) * a=a
$$

and

$$
a * \operatorname{anti}(a)=\operatorname{anti}(a) * a=\operatorname{neut}(a)
$$

The element $a$, neut $(a)$, and anti $(a)$ are collectively called a neutrosophic triplet and we denote it by $(a, n e u t(a)$, anti $(a))$. By neut $(a)$, we mean the neutral of $a$, and $a$ is just the first coordinate of a neutrosophic triplet and not a neutrosophic triplet [18].

For the same element " $a$ " in $N$, there may be more than one neutral neut(a) and more than one opposite $\operatorname{anti}(a)$. 
Definition 2. The element $b$ in $(N, *)$ is the second component, denoted by neut(.), of a neutrosophic triplet, if there exist other elements $a$ and $c$ in $N$ such that $a * b=b * a=a$ and $a * c=c * a=b$. The formed neutrosophic triplet is $(a, b, c)$ [12].

Definition 3. The element $c$ in $(N, *)$ is the third component, denoted by anti(.) of a neutrosophic triplet, if there exist other elements $a$ and $b$ in $N$ such that $a * b=b * a=a$ and $a * c=c * a=b$. The formed neutrosophic triplet is $(a, b, c)$ [12].

Definition 4. Let $(N, *)$ be a neutrosophic triplet set. Then $N$ is called a neutrosophic triplet group if the following conditions are satisfied [18].

1. If $(N, *)$ is well defined, i.e., for any $a, b \in N$, one has $a * b \in N$.

2. If $(N, *)$ is associative, i.e., $(a * b) * c=a *(b * c)$ for all $a, b, c \in N$.

The neutrosophic triplet group, in general, is not a group in the classical algebraic sense. We consider the neutrosophic neutrals as replacing the classical unitary element, and the neutrosophic opposites as replacing the classical inverse elements.

Example 1. Consider $\mathbb{Z}_{6}$ under multiplication modulo 6 , where $\mathbb{Z}_{6}=\{0,1,2,3,4,5\}$. Then the element 2 gives rise to a neutrosophic triplet because neut $(2)=4 \neq 1$, as $2 \times 4=4 \times 2=8 \equiv 2(\bmod 6)$. Also, anti(2) $=2$ because $2 \times 2=4$. Thus $(2,4,2)$ is a neutrosophic triplet. Similarly 4 gives rise to a neutrosophic triplet because neut $(4)=\operatorname{anti}(4)=4$. So $(4,4,4)$ is a neutrosophic triplet. Also, 3 gives rise to the neutrosophic triplet $(3,3,3)$. However, 3 has two neutrals: neut $(3)=\{3,5\}$, but 3 does not give rise to a neutrosophic triplet for neut $(3)=5$, since anti(3) does not exist in $\mathbb{Z}_{6}$ with respect to neut(3) $=5$. And lastly, 0 gives rise to a zero neutrosophic triplet as neut $(0)=$ anti $(0)=0$. The zero neutrosophic triplet is denoted by $(0,0,0)$.

The classical unitary element of $\mathbb{Z}_{6}$, with respect to multiplication modulo 6 , is 1 . The neutrosophic triplets on $\mathbb{Z}_{6}$ are: $(0,0,0),(0,0,2),(0,0,3),(0,0,4),(2,4,2),(3,33)$, and $(4,4,4)$.

$\mathbb{Z}_{6}$ is not a neutrosophic triplet set, since there is no neutrosophic triplet associated to the elements 1 and 5 from $\mathbb{Z}_{6}$, because: there is no neut $(1) \neq 1$, and respectively no neut $(5) \neq 1$.

But $M_{6}=\{0,2,3,4\} \subset \mathbb{Z}_{6}$ is a commutative neutrosophic group [18].

Example 2. Consider $\left(\mathbb{Z}_{10}, \#\right)$, where \# is defined as $a \# b=3 a b(\bmod 10)$.

Let $M_{10}=\{0,2,4,5,6,8\} \subset \mathbb{Z}_{10}$. Then $\left(M_{10}\right.$, \#) is a neutrosophic triplet group under the binary operation \# as shown in Table 1 [18].

Table 1. Cayley table of $\left(\mathbb{Z}_{10}, \#\right)$.

\begin{tabular}{lllllllllll}
\hline$\#$ & $\mathbf{0}$ & $\mathbf{1}$ & $\mathbf{2}$ & $\mathbf{3}$ & $\mathbf{4}$ & $\mathbf{5}$ & $\mathbf{6}$ & $\mathbf{7}$ & $\mathbf{8}$ & $\mathbf{9}$ \\
\hline $\mathbf{0}$ & 0 & 0 & 0 & 0 & 0 & 0 & 0 & 0 & 0 & 0 \\
$\mathbf{1}$ & 0 & 3 & 6 & 9 & 2 & 5 & 8 & 1 & 4 & 7 \\
$\mathbf{2}$ & 0 & 6 & 2 & 8 & 4 & 0 & 6 & 2 & 8 & 4 \\
$\mathbf{3}$ & 0 & 9 & 8 & 7 & 6 & 5 & 4 & 3 & 2 & 1 \\
$\mathbf{4}$ & 0 & 2 & 4 & 6 & 8 & 0 & 2 & 4 & 6 & 8 \\
$\mathbf{5}$ & 0 & 5 & 0 & 5 & 0 & 5 & 0 & 5 & 0 & 5 \\
$\mathbf{6}$ & 0 & 8 & 6 & 4 & 2 & 0 & 8 & 6 & 4 & 2 \\
$\mathbf{7}$ & 0 & 1 & 2 & 3 & 4 & 5 & 6 & 7 & 8 & 9 \\
$\mathbf{8}$ & 0 & 4 & 8 & 2 & 6 & 0 & 4 & 8 & 2 & 6 \\
$\mathbf{9}$ & 0 & 7 & 4 & 1 & 8 & 5 & 2 & 9 & 6 & 3 \\
\hline
\end{tabular}

It is also associative, i.e.,

$$
(a \# b) \# c=a \#(b \# c) .
$$


Now we take the LHS to prove the RHS.

$$
\begin{aligned}
(a \# b) \# c & =(3 a b) \# c \\
& =3(3 a b) c \\
& =9 a b c \\
& =3 a(3 b c) \\
& =3 a(b \# c) \\
& =a \#(b \# c)
\end{aligned}
$$

The classical unitary element on $\mathbb{Z}_{10}$ with respect to the law \# is $e=7$, since:

$$
a \# e=e \# a=3 a e=3 a(7)=21 a=a(\bmod 10) \text { for any } a \in \mathbb{Z}_{10}
$$

Therefore, we choose all triplets whose neutral elements are different from 7 , and we get the following neutrosophic triplets:

$(0,0,0),(0,0,2),(0,0,4),(0,0,5),(0,0,6),(0,0,8),(2,2,2),(4,2,6),(5,5,5),(6,2,4)$, and $(8,2,8)$.

All above neutrals neut(.) $=0,2$, and 5 are different from the classical unitary element 7 .

$Z_{10}$ is not a neutrosophic triplet group, nor even a neutrosophic triplet set.

But its subset $M_{10}=\{0,2,4,5,6,8\}$ is a commutative neutrosophic triplet group, since the law \# is well-defined, commutative, associative, and each element belonging to $M$ has a corresponding neutrosophic triplet.

\section{Neutrosophic Triplet Rings}

In this section, we introduce neutrosophic triplet rings and study some of their basic properties and notions.

Definition 5. Let $(N T R, *, \#)$ be a set together with two binary operations $*$ and \#. Then NTR is called a neutrosophic triplet ring if the following conditions hold:

1. $(N T R, *)$ is a commutative neutrosophic triplet group with respect to *;

2. (NTR,\#) is well defined and associateve;

3. $a \#(b * c)=(a \# b) *(a \# c)$ and $(b * c) \# a=(b \# a) *(c \# a)$ for all $a, b, c \in N T R$.

Notations 1. Since the neutrosophic triplet ring and the neutrosophic triplet field are algebraic structures endowed with two internal laws * and \#, in order to avoid any confusion, we use the following notation:

$n e u t^{*}(x)$ and $\operatorname{anti}^{*}(x)$ for the neutrals and anti's, respectively, of the element $x$ with respect to the law * and $n e u \#(x)$ and ant\# $(x)$ for the neutrals and anti's, respectively, of the element $x$ with respect to the law \#.

Remark 1. An NTR in general is not a classical ring.

Definition 6. Let $(N T R, *, \#)$ be a neutrosophic triplet ring and let $a \in N T R$. We call the structure a unitary neutrosophic triplet ring (UNTR) if each element a has a neut\#(a).

Definition 7. Let $(N T R, *, \#)$ be a neutrosophic triplet ring. NTR is called a commutative neutrosophic triplet ring if the law \# is commutative.

Definition 8. Let $(N T R, *, \#)$ be a neutrosophic triplet ring. If NTR has an element $b$ such that $a * b=b * a=b$ for all $a$ in NTR, then the element $b$ is called the zero neutrosophic element of NTR and denoted by 0.

Hence if NTR has 0 element then $0 * a=a^{*} 0=0$ for all $a$ in NTR. Also neut $*(0)=0$ and anti* $(0)=0$. 
Notations 2. For neutrosophic triplets with respect to the law * and \# are denoted by $(a, b, c)_{*}$ and $(a, b, c)_{\#}$ respectively.

Definition 9. Let $(N T R, *, \#)$ be a neutrosophic triplet ring and let $0 \neq a \in N T R$. If there exists a neutrosophic nonzero element $0 \neq b \in N T R$ such that $b \# a=0$, then $b$ is called a neutrosophic left zero divisor of $a$. Similarly, an element $0 \neq b \in N T R$ is called a neutrosophic right zero divisor of $0 \neq a \in N T R$ if $a \# b=0$.

A neutrosophic zero divisor of an element is one which is both a neutrosophic left zero divisor and a neutrosophic right zero divisor of that element.

Example 3. Let's consider the set $\left(\{a, b, c\},{ }^{*}, \#\right)$, where the laws are defined as follows.

Law * is well-defined, commutative (since its matrix into the Cayley Table is symmetric with respect to the main diagonal), and associative:

\begin{tabular}{|l|l|l|l|}
\hline$*$ & $a$ & $b$ & $c$ \\
$a$ & $a$ & $a$ & $a$ \\
\hline$b$ & $a$ & $b$ & $a$ \\
\hline$c$ & $a$ & $a$ & $c$ \\
\hline
\end{tabular}

Cayley Table for Law *

Let's prove the associativity: $x *(y * z)=\left(x^{*} y\right) * z$, for any $x, y, z \in\{a, b, c)$.

(1) If there is at least one " $a$ " among $x, y, z$, then the result is: $x^{*}(y * z)=a$ and $\left(x^{*} y\right) * z=a$, since " $a$ " transforms everything into " $a$ " according to the above table, i.e., $a^{*} a=a^{*} b=b^{*} a=a^{*} c=c^{*} a=a$.

(2) If there are only $b^{\prime}$, then $b^{*}\left(b^{*} b\right)=b$ and $(b * b) * b=b$.

(3) If there are only $c^{\prime}$, then $c^{*}\left(c^{*} c\right)=c$ and $\left(c^{*} c\right) * c=c$.

(4) If there are two $b^{\prime}$ s and one $c$, or two $c^{\prime}$ s and one $b$, then $x^{*}(y * z)=a$ and $\left(x^{*} y\right)^{*} z=a$,

Since $b$ multiplied $\left(^{*}\right)$ with $c$ will get $a\left(\right.$ or $\left.b{ }^{*} c=c{ }^{*} b=a\right)$ and further one " $a$ " transforms everything into a [as at point 1 above].

The neutrosophic triplets with respect to the law * are: $(a, a, a)_{*}(a, a, b)_{*}(a, a, c)_{*}(b, b, b)_{*},(c, c, c)_{*}$.

The law * has no classical unitary element.

Hence, $(\{a, b, c\}, *)$ is a commutative neutrosophic triplet group.

Law \# is well-defined, commutative (since its matrix into the below Cayley Table is symmetric with respect to the main diagonal), and associative:

\begin{tabular}{|l|l|l|l|}
\hline$\#$ & $a$ & $b$ & $c$ \\
$a$ & $a$ & $a$ & $a$ \\
\hline$b$ & $a$ & $a$ & $a$ \\
\hline$c$ & $a$ & $a$ & $a$ \\
\hline
\end{tabular}

Cayley Table for Law \#

Associativity is proved since for any $x, y, z \in\{a, b, c), x \#(y \# z)=a$ and $(x \# y) \# z=a$ because all multiplications (\#) give as result " $a$ ".

Let's prove the distributivity of \# with respect to *.

For any $x, y, z \in\{a, b, c), x \#(y * z)=a$, since the multiplication (\#) of anything gives " $a$ ", and $(x \# y) *(x$ $\# z)=a^{*} a=a$.

The set $(\{a, b, c\}, \#)$ is not a neutrosophic triplet set, since there is only one neutrosophic triplet $(a, a, a)\{w e$ have no corresponding neutrosophic triplet for $b$, nor for $c\}$, and consequently $(\{a, b, c\}$, \#) is not a neutrosophic triplet group.

The law \# has no classical unitary element. 
Hence, $\operatorname{NTR}=\left(\{a, b, c\},{ }^{*}, \#\right)$ is a commutative neutrosophic triplet ring, and not a neutrosophic triplet field.

Theorem 1. Let NTR be a commutative neutrosophic triplet ring and $a, b \in N T R$ such that $a, b$, neut\#( $a)$, neut\#(b), neut( $(a \# b)$, and anti\#( $a \# b)$ are cancellable and that neut\#( $a)$, neut\#(b) and anti\#( $(a)$, anti\#(b) do exist in NTR. Then

1. 1.neut $*(a) *$ neut $*(b)=$ neut $*(a * b)$,

2. anti* $(a) * \operatorname{anti} *(b)=\operatorname{anti} *(a * b)$,

3. neut\# $(a) \#$ neut\# $(b)=$ neut\# $(a \# b)$; and

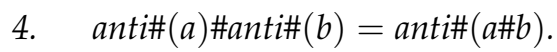

Proof. (1) and (2) are similar to the following.

(3) Consider the left-hand side, with neut\#(a)\#neut\#(b). Multiply by $a$ to the left and by $b$ to the right; then we have

$$
a \# n e u t^{\#}(a) \# \text { neut } t^{\#}(b) \# b=(a \# n e u t \#(a))^{\#}(\text { neut\#(b)\#b)=a\#b, }
$$

since \# is associative.

Now we consider the right-hand side; we have neut\#( $a \# b)$. Multiplying by $a$ to the left and by $b$ to the right, we have

$$
a \# n e u t^{\#}(a \# b) \# b=(a \# b) \# n e u t^{\#}(a \# b)=a \# b
$$

since \# is associative and commutative,

Thus, $L H S=(a \# b)=(a \# b) \#$ neut\# $(a \# b)=$ RHS.

(4) Considering the left-hand side, we have $\operatorname{anti}^{\#}(a) \# a n t i^{\#}(b)$.

Multiplying by $a$ to the left and by $b$ to the right, we have

$$
a \# a n t i^{\#}(a) \# a n t i i^{\#}(b) \# b=\left(a \# a n t i^{\#}(a)\right) \#\left(a n t i^{\#}(b) \# b\right)=(n e u t \#(a) \# n e u t \#(b))=n e u t \#(a \# b),
$$

using the previous proved result.

Now consider the right-hand side, where we have $a n t i^{\#}(a \# b)$.

Multiplying by $a$ to the left and by $b$ to the right, we have

$a \# a n t i \#(a \# b) \# b=(a \# b) \# a n t i \#(a \# b)=$ neut\# $(a \# b)$, since \# is associative and commutative.

\section{Open Question on Cancellability}

The "cancellability" condition in Theorem 1 is a heavy condition.

Using only commutativity and associativity of a law (without cancellability), it is sometimes possible to prove some neutrosophic triplet identities, but other times not.

In Example 3, the law ${ }^{*}$ does not satisfy the cancellability property, since $a * b=a{ }^{*} c$ but $b \neq c$ therefore $a$ is not cancellable, similarly $b^{*} a=b^{*} c$ but $a \neq c$ so $b$ is not cancellable, and $c^{*} a=c^{*} b$ but $b$ $\neq c$ therefore $c$ is not cancellable either, yet the law ${ }^{*}$ satisfies the first claim of the Theorem 1 :

$$
\text { neut } *(a) * \text { neut } *(b)=\text { neut } *(a * b)
$$

because

$$
\text { neut } *(a) * \text { neut } *(b)=a * b=a \text {, and neut } *(a * b)=\text { neut } *(a)=a
$$

but it does not satisfy the second claim of Theorem 1:

$$
\operatorname{anti} *(a) * \operatorname{anti} *(b)=\operatorname{anti} *(a * b)
$$


because

$$
\operatorname{anti} *(a) * \operatorname{anti} *(b)=\{a, b, c\} * b=\{a * b, b * b, c * b\}=\{a, b, a\}=\{a, b\}
$$

while anti* $(a * b)=\operatorname{anti}(a)=\{a, b, c\}$, since the element a has three anti's, resulted from the following neutrosophic triplets: $(a, a, a),(a, a, b),(a, a, c)$. But $\{a, b\} \neq\{a, b, c\}$.

Our open question is: When the cancellability property of the elements of a neutrosophic triplet set is needed, and when it is not, in order for the neutrosophic triplet identities to be valid?

Definition 10. Let $(N T R, *, \#)$ be a neutrosophic triplet ring and let $S$ be a subset of NTR. Then $S$ is called a neutrosophic triplet subring of NTR if $(S, *, \#)$ is a neutrosphic triplet ring.

Definition 11. Let $(N T R, *, \#)$ be a neutrosophic triplet ring and I be a subset of NTR. Then I is called a neutrosophic triplet ideal of NTR if the following conditions are satisfied.

1. $(I, *)$ is a neutrosophic triplet subgroup of $(N T R, *)$; and

2. For all $x \in I$ and $r \in N T R, x \# r \in I$ and $r \# x \in I$.

Theorem 2. Every neutrosophic triplet ideal is trivially a neutrosophic triplet subring, but the converse is not true in general.

Remark 2. Let $(N T R, *$ \#) be a neutrosophic triplet ring and let a $\in$ NTR. Then the following are true.

1. anti*(a) in general are not unique in NTR.

2. anti\#(a) (if they exist for some element a) in general are not unique in NTR.

Definition 12. Let $(N T R, *, \#)$ be a neutrosophic triplet ring and let $0 \neq a \in N T R$. Then $a$ is called a neutroosphic nilpotent element if $\left(a_{\#}\right)^{n}=0$, for some positive integer $n>1$, where $\left(a_{\#}\right)^{n}=\underbrace{\text { a\#a\#...\#a }}_{n}$ (a occurs $n$ times).

In the previous Example 3, one replaces $a=0, b=2, c=4$, then $\operatorname{NTR}_{2}=(\{0,2,4\}, *$, \#) is a commutative neutrosophic triplet ring, where $\left(2_{\#}\right)^{2}=2 \# 2=0$ with $2 \neq 0$, and $\left(4_{\#}\right)^{2}=4 \# 4=0$ with $4 \neq 0$, therefore the non-zero elements 2 and 4 are neutrosophic nilpotent elements in $N_{T} R_{2}$.

Also, since $2 \# 4=4 \# 2=0$, we say that 2 and 4 are neutrosophic zero-divisors in $N_{T} R_{2}$.

Theorem 3. Let NTR be a commutative neutrosophic triplet ring and a $\in$ NTR such that neut\#( $\left(a^{n}\right)$, and anti\# $\left(a^{n}\right)$ do exist in NTR for an integer $n \geq 1$. Let $a$, neut\#(a) be cancellable in NTR. Then

1. (neut\# $(a))^{n}=$ neut\# $\left(a^{n}\right)$,

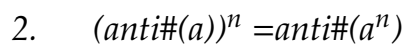

Proof. We prove by mathematical induction.

In Theorem 1(3), taking $a=b$, we have

$$
\text { neut\# }\left(a^{2}\right)=\text { neut\# }(a \# a)=\text { neut\# }(a) \# \text { neut\# }(a)=(\text { neut } \#(a))^{2} .
$$

We assume that our equality is true for any positive integer up to $n-1$, that is neut\# $\left(a^{n-1}\right)=(\text { neut } \#(a))^{n-1}$, and we need to prove it for $n$. By Theorem $1(3)$

$$
\text { neut } \#\left(a^{n}\right)=\text { neut }\left(a \# a^{n-1}\right)=\text { neut\#(a)\#neut } \#\left(a^{n-1}\right)=\operatorname{neut} \#(a) \#(\text { neut } \#(a))^{n-1}=(\text { neut } \#(a))^{n} .
$$

(2): similar to (1). 
Theorem 4. Let NTR be a commutative neutrosophic triplet ring and let $0 \neq a \in N T R$ such that neut\#( $\left.a^{k}\right)$, and anti\# $\left(a^{k}\right)$ do exist in NTR for an integer $k \geq 1$. If a is a neutrosophic nilpotent, that is, $a^{n}=0$ for some integer $n>$ 1 , then the following are true.

1. $(\text { neut\# }(a))^{n}=$ neut\# $(0) ;$ and

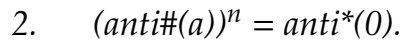

Proof. (1) Suppose that $0 \neq a$ is a neutrosophic nilpotent in NTR and let $\mathrm{a}^{\mathrm{n}}=0$ for some integer $\mathrm{n}>1$. Using Theorem 3(1) and (2), we have

$$
(\text { neut\# }(a))^{n}=\text { neut\# }\left(a^{n}\right)=\text { neut\# }(0) \text { and }(\operatorname{anti\# }(a))^{n}=\operatorname{anti\# }\left(a^{n}\right)=\operatorname{anti} \#(0) \text {. }
$$

\section{Integral Neutrosophic Triplet Domain and Neutrosophic Triplet Ring Homomorphism}

Section 4 is about the introduction of the integral neutrosophic triplet domain and some of its interesting properties. Moreover, in this section, we develop a neutrosophic triplet ring homomorphism.

Definition 13. A commutative neutrosophic triplet ringNTR is called an integral neutrosophic triplet domain if for all $a, b \in N T R, a \# b=0$ implies $a=0$ or $b=0$.

Theorem 5. Let NTR be an integral neutrosophic triplet domain. Then the following are true for all $a, b \in N T R$.

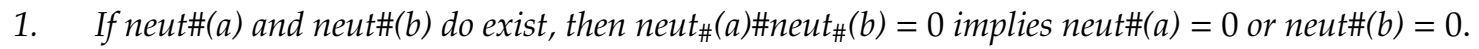

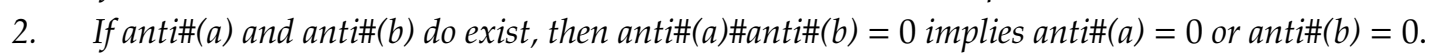

Proof. (1) Obvious, since NTR is an integral neutrosophic triplet domain, and $n e u t^{\#}(a)$ and $n e u t^{\#}(b)$ belong to NTR.

(2) Obvious, since NTR is an integral neutrosophic triplet domain, and anti\#(a) and anti\#(b) belong to NTR.

Definition 14. Let $\left(N T R_{1}, *, \#\right)$ and $\left(N T R_{2}, \oplus, \otimes\right)$ be two neutrosophic triplet rings. Let $f: N T R_{1} \rightarrow N T R_{2}$ be a mapping. Then $f$ is called a neutrosophic triplet ring homomorphism if the following conditions are true.

1. $f(a * b)=f(a) \oplus f(b)$, for all $a, b \in N T R_{1}$.

2. $f(a \# b)=f(a) \otimes f(b)$, for all $a, b \in N T R_{1}$.

3. $f\left(\right.$ neut $\left.^{*}(a)\right)=$ neut $^{\oplus}(f(a))$, for all $a \in N T R_{1}$.

4. $f\left(\operatorname{anti}^{*}(a)\right)=\operatorname{anti}^{\oplus}(f(a))$, for all $a \in N T R_{1}$.

\section{Neutrosophic Triplet Fields}

In this section, we study neutrosophic triplet fields and some of their interesting properties.

Definition 15. Let $(N T R, *, \#)$ be a neutrosophic triplet set together with two binary operations * and \#. Then $(N T R, *, \#)$ is called a neutrosophic triplet field if the following conditions hold.

1. $(N T R, *)$ is a commutative neutrosophic triplet group with respect to *.

2. (NTR, \#) is a neutrosophic triplet group with respect to \#.

3. $a \#(b * c)=(a \# b) *(a \# c)$ and $(b * c) \# a=(b \# a) *(c \# a)$ for all $a, b, c \in N T R$. 
Example 4. $M_{6}=\{0,2,3,4\} \subset \mathbb{Z}_{6}$ is a commutative neutrosophic group under multiplication law \# modulo 6 (Example 1). The Cayley Table for Law \# is as the following:

\begin{tabular}{l|llll}
$\#$ & 0 & 2 & 3 & 4 \\
\hline 0 & 0 & 0 & 0 & 0 \\
2 & 0 & 4 & 0 & 2 \\
3 & 0 & 0 & 3 & 0 \\
4 & 0 & 2 & 0 & 4
\end{tabular}

Now we define a law * for the set $M_{6}$ as the following table:

\begin{tabular}{l|llll}
$*$ & 0 & 2 & 3 & 4 \\
\hline 0 & 0 & 0 & 0 & 0 \\
2 & 0 & 2 & 0 & 0 \\
3 & 0 & 0 & 3 & 0 \\
4 & 0 & 0 & 0 & 4
\end{tabular}

It is easily seen that $(0,0,0)_{*}(2,2,2)_{*}(3,3,3)_{*}$ and $(4,4,4)_{*}$ are neutrosophic triplets, the law ${ }^{*}$ is well defined and commutative. Let us prove its associative:

If $a=b=c=0$ then $a *(b * c)=0=(a * b) * c$.

If $a=b=c=2$ then $a *(b * c)=2=\left(a^{*} b\right) * c$.

If $a=b=c=3$ then $a *(b * c)=3=(a * b) * c$.

If $a=b=c=4$ then $a *(b * c)=4=\left(a^{*} b\right) * c$.

If at least two of $a, b$ and $c$ are different, we have $a *(b * c)=0=(a * b) * c$.

So associative law for ${ }^{*}$ is true. Hence $M_{6}$ is a commutative neutrosophic group under the law ${ }^{*}$.

Also it is easily check that $a \#\left(b{ }^{*} c\right)=(a \# b) *(a \# c)$ for all $\mathrm{a}, \mathrm{b}$ and $\mathrm{c}$ in $M_{5}$ is satisfied. (Note that * and \# are commutative). So $\left(M_{5}{ }^{*}, \#\right)$ is NTF.

Proposition 1. A neutrosophic triplet field NTF always has an anti(a) for every a $\in$ NTF with respect to both laws * and \#.

Proof. The proof is straightforward.

Theorem 6. A neutrosophic triplet ring is not in general a neutrosophic triplet field.

Counterexample is done in Example 3.

Theorem 7. A neutrosophic triplet field NTF is not in general an integral neutrosophic triplet domain INTD.

Theorem 8. Assume that $f: N T R_{1} \rightarrow N T R_{2}$ is a neutrosophic triplet ring homomorphism. The following then hold.

1. If $S$ is a neutrosophic triplet subring $N T R_{1}(*, \#)$, then $f(S)$ is a neutrosophic triplet subring of $N T R_{2}(\oplus, \otimes)$.

2. If $U$ is a neutrosophic triplet subring of $N T R_{2}$, then $f^{-1}(U)$ is a neutrosophic triplet subring of $N T R_{1}$.

3. If I is a neutrosophic triplet ideal of $N T R_{2}$, then $f^{-1}(I)$ is a neutrosophic triplet ideal of $N T R_{1}$.

4. If $f$ is onto, and $J$ is an ideal of $N T R_{1}$, then $f(j)$ is an ideal of $N T R_{2}$.

Proof. (1) Let $a, b \in S$, then $\mathrm{a}^{*} \mathrm{~b} \in \mathrm{S}$, neut ${ }^{*}(\mathrm{a}) \in \mathrm{S}$, anti*(a) $\in \mathrm{S}$. Then $f(a), f(b) \in f(S)$ and $f(a * b) \in f(S)$, but $f(a * b)=f(a) \oplus f(b)$, since $f$ is a homomorphism. Thus, we have proved that if $f(a), f(b) \in f(S)$, then $f(a) \oplus f(b) \in f(S$. Since neut*(a) and anti*(a) $\in S, f($ neut $(a))$ and $f(\operatorname{anti}(a)) \in f(S)$ since $f$ is a 
homomorphism. But $\mathrm{f}\left(\right.$ neut $\left.^{*}(\mathrm{a})\right)=$ neut $^{\oplus} \mathrm{f}(\mathrm{a})$, and $\mathrm{f}\left(\operatorname{anti}^{*}(\mathrm{a})\right)=\operatorname{anti}^{\oplus} \mathrm{f}(\mathrm{a})$. Therefore, if $f(a) \in f(S)$, then neut $^{\oplus} \mathrm{f}(\mathrm{a})=\mathrm{f}\left(\right.$ neut $\left.^{*}(\mathrm{a})\right) \in f(S)$ and, similarly,

$$
\operatorname{anti}^{\oplus} \mathrm{f}(\mathrm{a})=\mathrm{f}(\operatorname{anti} *(\mathrm{a})) \in f(S) .
$$

Now, if $a, b \in S$, then $a \# b \in S$. Since $a \# b \in S, f(a \# b) \in f(S)$. But $f(a \# b)=f(a) \oplus f(b)$. Therefore, if $f(a), f(b) \in S$, then $f(a) \otimes f(b)=f(a \# b)=f(S)$.

(2) $f^{-1}(U)=\left\{a \in N T R_{1}: f(a) \in U\right\}$. Let $\mathrm{a}, \mathrm{b} \in f^{-1}(U)$. Then $f(a), f(b) \in U . f(a) \oplus f(b)=f\left(a^{*} b\right)$ $\in \mathrm{U}$ and $f(a) \otimes f(b)=f(a \# b) \in \mathrm{U}$. Then: $a^{*} b, a \# b \in f^{-1}(U)$. Since $a \in f^{-1}(U)$, then $\mathrm{f}(\mathrm{a}) \in \mathrm{U}$. But also neut $t_{\oplus}(f(a)) \in U$, anti $i_{\oplus}(f(a)) \in U$, because $U$ is a neutrosophic subring of NTR 2 and $f$ is a neutro-homomorphism. But neut $t_{\oplus}(f(a))=f\left(\right.$ neut $\left._{*}(a)\right) \in U$, anti ${ }_{\oplus}(f(a))=f\left(\right.$ anti $\left._{*}(a)\right) \in U$, whence neut $_{*}(a) \in f^{-1}(U)$, anti $_{*}(a) \in f^{-1}(U)$.

(3) $f^{-1}(I)=\left\{a \in N T R_{1}: f(a) \in I\right\}$. Let $a \in f^{-1}(I)$ and $r \in N T R_{1}$. Then $f(a) \in I$ and $f(r) \in N T R_{2}$. Because $f$ is a neutro-homomorphism and $I$ an ideal of $\mathrm{NTR}_{2}$, one has:

$$
f(a) \otimes f(r)=f(a \# r) \in I,
$$

hence $a \# r \in f^{-1}(I)$.

(4) Let $j \in f(J)$ and $r \in N T R_{2}$. Since $f$ is onto, then $\exists h \in J \subset N T R_{1}$ such that $f(h)=j$ and $\exists s \in N T R_{1}$ such that $f(s)=r$. h\#s $\in J$ because $\mathrm{J}$ is an ideal of $N T R_{1}$. Then:

$$
f(h \# s)=f(h) \otimes f(s)=j \otimes s \in f(J)
$$

which is true, since $h \in J$, which is an ideal in $N T R_{1}$, while $s \in N T R_{1}$.

\section{Conclusions}

In this paper, we presented the neutrosophic triplet ring. Further, we presented the zero divisor, neutrosophic triplet subring, neutrosophic triplet ideal, nilpotent, integral neutrosophic triplet domain, and neutrosophic triplet ring homomorphism. Finally, we presented the neutrosophic triplet field. In the future, we can develop neutrosophic triplet vector spaces, neutrosophic modules, and neutrosophic triplet near rings, and so on.

Author Contributions: M.A. defined and studied the properties of neutrosophic triplet rings. F.S. defined and studied the properties of the neutrosophic triplet fields. M.K. provided examples and the neutrosophic triplet ring homomorphism and organized the paper.

Acknowledgments: The authors acknowledge that there is no conflict of interest. Further, funding is not available for this work.

Conflicts of Interest: The authors declare no conflict of interest.

\section{References}

1. Kleiner, I. From Numbers to Ring: The Early History of Ring Theory. In Elemente der Mathematik; Springer: Berlin/Heidelberg, Germany, 1998; Volume 53, pp. 18-35.

2. Conferences of the Mathematics and Statistics Department of the Technical University of Catalonia. Emmy Noether Course. Available online: https:/upcommons.upc.edu/bitstream/handle/2117/81399/CFME-vol-6. pdf? sequence=1\&isAllowed =y (accessed on 21 March 2018).

3. Connes, A. Introduction to non-commutative differential geometry. In Lectures Notes in Physics; Springer: Berlin/Heidelberg, Germany, 1984; Volume 1111, pp. 3-16.

4. Connes, A. Non-commutative differential geometry. In Publications Mathematics; Springer: Berlin/Heidelberg, Germany, 1985; Volume 62, pp. 257-360.

5. Connes, A. The action functional in non-commutative geometry. In Communications in Mathematical Physics; Springer: Berlin/Heidelberg, Germany, 1988; Volume 11, pp. 673-683. 
6. Kaplansky, I. An Introduction to the Differential Algebra; Hermann: Paris, France, 1957.

7. Kaplansky, I. Fields and Rings, 2nd ed.; The University of Chicago Press: Chicago, IL, USA, 1972; ISBN 0-226-42450-2.

8. Herstein, I. Wedderburn's theorem and a theorem of Jacobson. Am. Math. Mon. 1961, 68, 249-251. [CrossRef]

9. Smarandache, F. Neutrosophy: Neutrosophic Probability, Set, and Logic; ProQuest Information \& Learning: Ann Arbor, MI, USA, 1998; 105p, Available online: http://fs.gallup.unm.edu/eBook-neutrosophics6.pdf (accessed on 21 March 2018).

10. Atanassov, K.T. Intuitionistic fuzzy sets. Fuzzy Sets Syst. 1986, 20, 87-96. [CrossRef]

11. Zadeh, L.A. Fuzzy sets. In Fuzzy Sets, Fuzzy Logic and Fuzzy Systems: Selected Papers by Lotfi A. Zadeh; World Scientific: River Edge, NJ, USA, 1996; pp. 394-432.

12. Kandasamy, W.B.V.; Smarandache, F. Some Neutrosophic Algebraic Structures and Neutrosophic N-Algebraic Structures; Hexis: Frontigan, France, 2006; p. 219.

13. Kandasamy, W.B.V.; Smarandache, F. N-Algebraic Structures and S-N-Algebraic Structures; Hexis: Phoenix, AZ, USA, 2006; p. 209.

14. Kandasamy, W.B.V.; Smarandache, F. Basic Neutrosophic Algebraic Structures and Their Applications to Fuzzy and Neutrosophic Models; Hexis: Frontigan, France, 2004; p. 149.

15. Ali, M.; Smarandache, F.; Shabir, M.; Vladareanu, L. Generalization of Neutrosophic Rings and Neutrosophic Fields. Neutrosophic Sets Syst. 2014, 5, 9-14.

16. Agboola, A.; Akinleye, S. Neutrosophic Vector Spaces. Neutrosophic Sets Syst. 2014, 4, 9-18.

17. Agboola, A.; Akwu, A.; Oyebo, Y. Neutrosophic groups and subgroups. Int. J. Math. Comb. 2012, 3, 1.

18. Smarandache, F.; Ali, M. Neutrosophic triplet group. Neural Comput. Appl. 2018, 29, 595-601. [CrossRef]

(C) 2018 by the authors. Licensee MDPI, Basel, Switzerland. This article is an open access article distributed under the terms and conditions of the Creative Commons Attribution (CC BY) license (http://creativecommons.org/licenses/by/4.0/). 